\title{
99m Tc-MIBI Imaging in the Presurgical Characterization of Thyroid Follicular Neoplasms: Relationship to Multidrug Resistance Protein Expression
}

Enrico Saggiorato ${ }^{1}$, Tiziana Angusti ${ }^{2}$, Rosy Rosas $^{3}$, Morena Martinese ${ }^{1}$, Monica Finessi ${ }^{1}$, Federico Arecco ${ }^{1}$, Edoardo Trevisiol ${ }^{2}$, Nicoletta Bergero ${ }^{4}$, Barbara Puligheddu ${ }^{1}$, Marco Volante $^{3}$, Valerio Podio ${ }^{2}$, Mauro Papotti ${ }^{3}$, and Fabio Orlandi ${ }^{1}$

${ }^{I}$ Section of Endocrinology, Division of Internal Medicine, Department of Clinical and Biological Sciences, Gradenigo Hospital, University of Turin, Turin, Italy; ${ }^{2}$ Division of Nuclear Medicine, Department of Clinical and Biological Sciences, San Luigi Hospital, University of Turin, Turin, Italy; ${ }^{3}$ Division of Pathology, Department of Clinical and Biological Sciences, San Luigi Hospital, University of Turin, Turin, Italy; and ${ }^{4}$ Department of Anesthesiology and Intensive Care, San Giovanni Battista Hospital, University of Turin, Turin, Italy

Recently, thyroid $99 \mathrm{~m}$ Tc-methoxyisobutylisonitrile (99mTc-MIBI) scintiscanning has been proposed in an attempt to preoperatively identify thyroid malignancies, but discrepant results have been reported for oncocytic lesions. The aim of this study was to investigate the usefulness of visual and semiquantitative analyses of ${ }^{99 \mathrm{~m} T c-M I B I}$ scintigraphy for preoperatively characterizing thyroid nodules with indeterminate cytologic diagnoses, segregating in advance nononcocytic variants from those that are oncocytic. This study also aimed to analyze the relationship between $99 \mathrm{mTc}-\mathrm{MIBI}$ images and P-glycoprotein (P-gp)/multidrug resistance-associated protein-1 (MRP1) immunohistochemical expression. Methods: Fifty-one consecutive patients with cold thyroid nodules cytologically diagnosed as nononcocytic or oncocytic follicular neoplasm were prospectively studied. Visual and semiquantitative 99mTc-MIBI scanning was performed and the diagnoses of the lesions were histologically proven by subsequent thyroidectomy. Immunohistochemical evaluation of P-gp and MRP1 was also performed on surgical samples. Results: Visual and semiquantitative ${ }^{99 m}$ Tc-MIBI scintiscans showed a low specificity in preoperatively discriminating malignant oncocytic lesions. In nononcocytic nodules, the semiquantitative method was more accurate than the visual $(94.44 \%$ and $77.78 \%$, respectively). P-gp protein expression was negative in all thyroid lesions, whereas apical plasma membrane MRP1 expression was found in $78 \%$ of the lesions with a negative ${ }^{99 m}$ Tc-MIBI retention index, compared with $11 \%$ of lesions with a positive retention index, correlating most strongly with a negative ${ }^{99 m}$ Tc-MIBI RI in those cases with strong MRP1 apical expression. Conclusion: Semiquantitative ${ }^{99 m}$ Tc-MIBI scintigraphy is an adjunctive method to predict preoperatively the malignant behavior of nononcocytic follicular thyroid nodules indeterminate at fine-needle aspiration biopsy, with a potential

Received Apr. 6, 2009; revision accepted Jul. 28, 2009.

For correspondence or reprints contact: Enrico Saggiorato, Section of Endocrinology, Division of Internal Medicine, Department of Clinical and Biological Sciences, Gradenigo Hospital, University of Turin, c.so Regina

Margherita 10, 10153 Turin (TO), Italy.

E-mail: enrico.saggiorato@unito.it

COPYRIGHT ( 2009 by the Society of Nuclear Medicine, Inc. impact on the definition of their clinical management. Moreover, the good correlation found between immunohistochemical apical expression of MRP1 and the scintigraphic findings supports the ${ }^{99 m}$ Tc-MIBI results and provides tissue information on the molecular mechanisms responsible for ${ }^{99 m T c-M I B I}$ images in thyroid lesions.

Key Words: thyroid follicular neoplasms; thyroid ${ }^{99 m} \mathrm{Tc}-\mathrm{MIBI}$ scintigraphy; fine-needle aspiration biopsy; P-glycoprotein; multidrug resistance-associated protein-1

J Nucl Med 2009; 50:1785-1793

DOI: 10.2967/jnumed.109.064980

$\mathbf{T}$ he diagnostic approach to thyroid nodules is usually based on fine-needle aspiration biopsy (FNAB), a wellestablished complement to clinical and ultrasound examinations (1). However, the most important FNAB limitation is the lack of sensitivity in the characterization of follicular neoplasms because of its inability to detect capsular invasion and vessel infiltration of the tumor $(2,3)$.

An additional FNAB diagnostic restraint is represented by some cases of follicular variants of papillary carcinoma in which the classic diagnostic cytologic criteria of papillary carcinoma are lacking or not completely unequivocal $(4,5)$, as well as by some cases of microfollicular goiter with a hypercellular cytologic pattern; thus, these lesions are often cytologically interpreted as follicular neoplasms (6).

Although immunocytochemical evaluation of some tumorassociated antigens, such as galectin-3 (7-14), cell surface mesothelial antigen HBME-1 $(8,14-17)$, and cytokeratin-19 $(8,16,17)$, was recently successfully introduced for the thyroid to improve preoperative cytologic diagnosis, the issue of follicular neoplasm remains incompletely solved 
and these lesions still need surgical procedures for a definitive diagnosis.

Hence, the identification of new diagnostic approaches is of paramount importance to provide reliable criteria for malignancy preoperatively when FNAB findings are indeterminate.

To this purpose, some investigators have proposed thy-

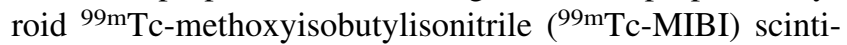
scanning in an attempt to differentiate benign from malignant thyroid nodules (18-24). Particularly, differentiated thyroid carcinoma has been reported to intensively accumulate and persistently retain ${ }^{99 m}$ Tc-MIBI (18-24). However, discrepant results have been reported about the usefulness of ${ }^{99 \mathrm{~m}}$ Tc-MIBI scanning in identifying thyroid carcinoma, especially in mitochondria-rich oncocytic lesions $(22,25-27)$.

The mechanism of cellular radiopharmaceutical accumulation has been reported to depend on the size of a tumor, the blood flow within it (28), and the richness of mitochondria in the tumor cells $(28,29)$. MIBI reversibly passes into the cytoplasm via thermodynamic driving forces and irreversibly passes into the mitochondria using the electrical gradient generated by a high negative inner transmembrane mitochondrial potential (28-30). Accordingly, more intense MIBI concentrations have been found in malignant tumor cells than in normal cells because of the higher electrical gradient of the former (28).

In addition, ${ }^{99 \mathrm{~m}} \mathrm{Tc}-\mathrm{MIBI}$ has been shown to be a substrate for the MDR1 gene coded P-glycoprotein (P-gp) (28,31-33) and multidrug resistance-associated protein-1 (MRP1) (3235 ) efflux pumps, supporting the rapid ${ }^{99 \mathrm{~m}} \mathrm{Tc}-\mathrm{MIBI}$ washout displayed by tumor cells expressing high levels of P-gp or MRP1 and the delayed washout displayed by cells with undetectable levels or altered P-gp or MRP1.

The aim of this study was to investigate the usefulness of qualitative and semiquantitative analyses of dual-phase ${ }^{99 m}$ Tc-MIBI thyroid scintigraphy for differentiating benign from malignant thyroid nodules, separately, in patients with a cytologic diagnosis of nononcocytic or oncocytic follicular neoplasms, which are in advance distinguished by fineneedle aspiration cytomorphologic evaluation. In addition, we analyzed the relationship between scintigraphic ${ }^{99 \mathrm{~m}} \mathrm{Tc}-$ MIBI findings and the expression of P-gp and MRP1 in the corresponding thyroid nodules.

\section{MATERIALS AND METHODS}

\section{Patients}

This study prospectively included 51 consecutive patients (11 men and 40 women; age range, 28-72 y; median, 55 y) with ${ }^{99 \mathrm{~m}} \mathrm{Tc}$-pertechnetate-cold solitary (or prevalent) thyroid nodules at least $10 \mathrm{~mm}$ in diameter (median, $17 \mathrm{~mm}$; range, 10-80 mm), cytologically diagnosed as Thy3 (nononcocytic or oncocytic follicular neoplasm) according to the British Thyroid Association guidelines (Table 1) (36). All patients were diagnosed and treated at San Luigi Hospital, Orbassano (Turin, Italy), between 2006 and 2007.
In all patients, serum levels of free thyroxine, free triiodothyronine, thyroid-stimulating hormone, antithyroglobulin and antithyroid peroxidase antibodies, parathyroid hormone, and calcitonin were determined. All patients underwent a thyroid ultrasound examination followed by ${ }^{99 \mathrm{~m} T c-M I B I}$ imaging and were subsequently operated on to confirm or exclude a malignant lesion.

The study was approved by the San Luigi Hospital review board, and written informed consent was obtained from each patient.

\section{Fine-Needle Aspiration Biopsy}

Preoperative FNAB was performed with a 22-gauge needle attached to a 30-mL plastic syringe. The aspirated fluid was in part expelled and smeared onto charged slides and was fixed and stained with a rapid hematoxylin-and-eosin method for adequacy evaluation or processed for Giemsa staining. The remaining material was used to prepare alcohol-fixed cell blocks and hematoxylin- and eosin-stained sections (9).

Oncocytic follicular neoplasms were defined by the presence of oncocytic cells-large cells characterized by abundant deeply eosinophilic and granular cytoplasm, with hyperchromatic nuclei having prominent nucleoli (37) - in at least $50 \%$ of the total follicular cells present on smears or cell-block sections.

\section{Cytologic and Histologic Samples}

The histologic diagnoses of the 36 nononcocytic follicular neoplasms were microfollicular goiter (9 cases), follicular adenoma (12 cases), minimally invasive follicular carcinoma (2 cases), follicular variants of papillary carcinoma (12 cases), and poorly differentiated carcinoma (1 case) (Table 1). The histologic diagnoses of the 15 oncocytic follicular neoplasms were oncocytic microfollicular goiter (5 cases), oncocytic follicular adenoma (6 cases), oncocytic insular carcinoma (1 case), and oncocytic follicular variants of papillary carcinoma (3 cases) (Table 1).

Thyroid tumors were classified according to the most recent criteria of the World Health Organization (37). Oncocytic variants of follicular adenoma, follicular variants of papillary carcinoma, insular carcinoma, and microfollicular goiter were defined by the presence of oncocytic changes in at least $75 \%$ of the lesion (37).

\section{Thyroid 99mTc-MIBI Scintigraphy}

99mTc-MIBI scintigraphy was performed on each patient using a large-field-of-view $\gamma$-camera (Axis; Philips Netherland) equipped with a high-resolution parallel-hole low-energy collimator. Static images of the neck were acquired in the anterior view with a $128 \times 128$ matrix, a 1.33 zoom, and a pixel size of $1.75 \mathrm{~mm}$ and stored on a Philips Odyssey computer. Images were obtained at 10 min (early image) and $120 \mathrm{~min}$ (late image) after intravenous injection of $400 \mathrm{MBq}$ of ${ }^{99 \mathrm{~m}} \mathrm{Tc}-\mathrm{MIBI}$, according to American guidelines (38). Labeling efficiency was assessed by thin-layer chromatography and was found always to exceed $94 \%$. To ensure correct positioning of the patients, we always included both salivary glands and activity from the myocardium in the ${ }^{99 \mathrm{~m}} \mathrm{Tc}-$ MIBI images.

The images were independently assessed by 2 experienced nuclear physicians before surgery. They used both a visual scoring method and a semiquantitative technique and were not aware of 


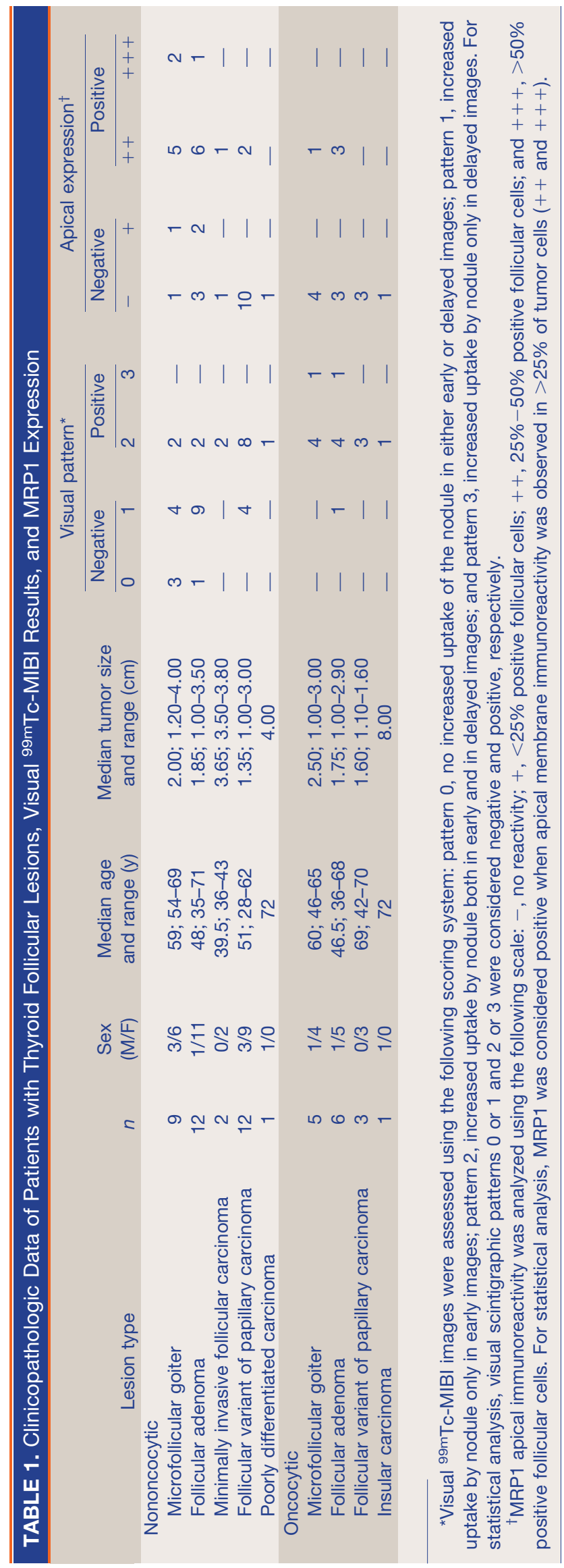

the clinical data or histologic results. If they disagreed, they discussed the case and reached a consensus.

\section{Visual Analysis of Images}

The early and delayed ${ }^{99 m}$ Tc-MIBI thyroid images of a given patient were placed side by side for comparison. The visual assessment used the following scoring system developed in our institution: pattern 0 , no increased uptake of the nodule in either early or delayed images; pattern 1, increased uptake of the nodule only in early images; pattern 2, increased uptake of the nodule both in early and in delayed images; and pattern 3, increased uptake of the nodule only in delayed images.

\section{Semiquantitative Analysis of Images}

Both early and delayed images were displayed on the same screen. The pertechnetate image of the patient was also displayed on the same screen when definite anatomic landmarks were needed. On each early image, an exact region of interest was drawn over the perimeter of the thyroid nodule and then moved to the opposite normal lobe. These regions of interest were then copied to the delayed images. If high radiotracer uptake in the nodule made it difficult to see the contralateral normal lobe, the intensity of the image was increased until the normal lobe was clearly visualized and the pertechnetate image was used as an anatomic landmark. For background, early-image rectangular regions of interest were drawn on the right superior region of the patient's thorax and then copied to the delayed images. The mean count in each region of interest was determined, and early ratio (ER) and delayed ratio (DR) were calculated by dividing nodule counts by normal-tissue counts after area correction for background activity. The retention index (RI) was then found using the formula RI $=(D R-E R) \times 100 / E R$.

\section{Antibodies}

Two primary monoclonal antibodies were used in this study: clone C219 diluted 1/150 (Zymed Laboratories) to P-gp and clone QCRL-1 diluted 1/400 (Sigma-Aldrich) to MRP1. These antibodies were selected from a preliminary pilot immunohistochemical study involving a panel of commercial standardized antibodies.

\section{Immunoperoxidase Technique}

Paraffin blocks containing surgical specimens were cut at a $4-\mu \mathrm{m}$ thickness, collected onto poly-L-lysine-coated slides, dewaxed in xylene, rehydrated in decreasing ethanol concentrations, and incubated for $10 \mathrm{~min}$ in phosphate-buffered saline (PBS) (pH 7.4). A heat-induced antigen retrieval procedure was performed for all antibodies by placing slides in $0.01 \mathrm{M}$ ethylene-diaminetetraacetate buffer ( $\mathrm{pH} 8.0)$ in a microwave oven set at high power for 3 consecutive cycles of $5 \mathrm{~min}$ each. The slides were left to cool for $20 \mathrm{~min}$ at room temperature and rinsed in PBS. Endogenous peroxidase activity was quenched with methanol-hydrogen peroxide $3 \%$ for $15 \mathrm{~min}$ at room temperature. Slides were then washed twice in PBS for $5 \mathrm{~min}$, and tissue sections were incubated first in the blocking solution (ChemMate Buffer Kit; DakoCytomation) and then with the primary antibodies in a humidified chamber at room temperature for $45 \mathrm{~min}$. After a prolonged wash in PBS, the sections were incubated with an enzyme-labeled polymer preconjugated with antimouse secondary antibodies (Envision System; DakoCytomation) at room temperature for 30 min. The sections were finally washed 3 times in PBS and incubated with $3^{\prime}-3^{\prime}$-diaminobenzidine-tetrahydrochloride for 10 
min. Slides were subsequently rinsed in tap water, counterstained with Mayer hemalum solution, mounted in Entellan (Merck), and examined with a DM-RBE photomicroscope (Leica Microsystems AG). Positive controls were human liver, kidney, and colon tissues. Negative controls were obtained by omitting the primary antibody.

P-gp and MRP1 immunostaining was evaluated by 3 independent observers without knowledge of clinical and pathologic data. MRP1 topographic expression (cytoplasmic or apical membranous immunoreactivity) was analyzed using the following semiquantitative scale: - (no reactivity), + (focal reactivity, $<25 \%$ of positive follicular cells), ++ (moderate reactivity, $25 \%-50 \%$ of positive follicular cells), and +++ (diffuse reactivity, $>50 \%$ of positive follicular cells).

\section{Statistical Analysis}

Visual scintigraphic patterns 2 or 3 and 0 or 1 were considered positive and negative, respectively.

The intra- and interobserver variability (reproducibility) and repeatability coefficients (39) were computed for both ER and DR measures.

The Mann-Whitney $U$ test (Statistica software, version 6.1; Statsoft Italia s.r.l.) was used to determine the differences in ER, DR, and RI indices between benign and malignant nononcocytic lesions, as well as between benign and malignant oncocytic lesions. Results were considered significant when the $P$ value was less than 0.05 . The histologic diagnosis on the surgical specimens was regarded as the gold standard.

The correlation between nodule size and RI value was expressed using the Spearman rank correlation coefficient $(s)$ with 95\% confidence interval $(\mathrm{CI})$ and $P$ value (level of significance, $P<0.05)$.

Receiver operating characteristic analysis (MedCalc software, version 10.0.2.0; Frank Shoonjans) was performed to determine the RI threshold above which malignant nononcocytic thyroid nodules could be detected.

Sensitivity, specificity, positive and negative predictive values, likelihood ratio, and diagnostic accuracy of visual and semiquantitative ${ }^{99 \mathrm{~m}} \mathrm{Tc}-\mathrm{MIBI}$ analyses were computed for benign versus malignant nononcocytic and oncocytic lesions, separately.

For the purpose of statistical analysis, MRP1 was considered positive when apical membrane immunoreactivity was observed in more than $25 \%$ of tumor cells.

MRP1 immunohistochemistry was associated and correlated to ${ }^{99 \mathrm{~m} T c-M I B I}$ findings by means of nonparametric 2-tailed Fisher exact testing and Kruskal-Wallis 1-way ANOVA (level of significance, $P<0.01)$.

\section{RESULTS}

The results of the clinicopathologic findings, visual analysis of ${ }^{99 \mathrm{~m}} \mathrm{Tc}-\mathrm{MIBI}$ images, and MRP1 expression are summarized in Table 1.

All patients had normal values for thyroid-stimulating hormone and parathyroid hormone and negative calcitonin levels.

No significant difference in nodule size was found between benign and malignant thyroid lesions $(P=0.70)$ or between positive and negative RI values $(P=0.33)$ of the nodules.

\section{Visual Analysis}

Eleven of 15 nononcocytic carcinomas showed a significantly increased ${ }^{99} \mathrm{~m}$ Tc-MIBI uptake at $120 \mathrm{~min}$ (scintigraphic pattern 2), whereas 4 follicular variants of papillary carcinoma presented a total radiotracer washout at $120 \mathrm{~min}$ (Table 1). Among nononcocytic benign lesions, 17 of 21 samples displayed a ${ }^{99 \mathrm{~m}}$ Tc-MIBI pattern 0 or 1 , whereas 4 cases ( 2 follicular adenomas and 2 micofollicular goiters) showed a pattern 2 (Table 1).

The sensitivity and specificity of ${ }^{99 \mathrm{~m}} \mathrm{Tc}-\mathrm{MIBI}$ visual analysis in differentiating benign from malignant nononcocytic tumors were $73.33 \%$ (CI, 44.91-92.05) and 80.95\% (CI, 58.08-94.44), respectively. Positive and negative predictive values were $73.33 \%$ (CI, 44.91-92.05) and 80.95\% (CI, 58.08-94.44), respectively. The positive likelihood ratio was 3.85 (CI, 1.51-9.79). The diagnostic accuracy of $99 \mathrm{~m}$ Tc-MIBI visual analysis was $77.78 \%$ (Table 2).

All but 1 oncocytic lesion exhibited a significantly increased ${ }^{99 \mathrm{~m}}$ Tc-MIBI uptake at $120 \mathrm{~min}$ (scintigraphic pattern 2 or 3 ) (Table 1 ).

The sensitivity and specificity of ${ }^{99} \mathrm{~m}$ Tc-MIBI visual analysis in differentiating benign from malignant oncocytic tumors were $100 \%$ (CI, 40.23-100) and 9.09\% (CI, 1.5141.33), respectively. Positive and negative predictive values were $28.57 \%$ (CI, 8.57-58.08) and 100\% (CI, 16.55-100), respectively. The positive likelihood ratio was 1.10 (CI, 0.91-1.33). The diagnostic accuracy of ${ }^{99 \mathrm{~m}} \mathrm{Tc}-\mathrm{MIBI}$ visual analysis was $33.33 \%$.

\section{Semiquantitative Analysis}

The results of semiquantitative ${ }^{99 \mathrm{~m}} \mathrm{Tc}-\mathrm{MIBI}$ analysis are reported in Table 3 .

\section{TABLE 2. Discrimination Between Malignant and Benign Nononcocytic Thyroid Lesions by ${ }^{99 m}$ Tc-MIBI Analyses}

\begin{tabular}{|c|c|c|c|c|c|c|}
\hline $\begin{array}{l}\text { Type of } \\
\text { analysis }\end{array}$ & $\begin{array}{c}\text { Sensitivity } \\
(\%)\end{array}$ & $\begin{array}{c}\text { Specificity } \\
(\%)\end{array}$ & $\begin{array}{c}\text { Positive predictive } \\
\text { value (\%) }\end{array}$ & $\begin{array}{c}\text { Negative predictive } \\
\text { value }\end{array}$ & $\begin{array}{c}\text { Positive } \\
\text { likelihood ratio }\end{array}$ & Accuracy \\
\hline Visual & $\begin{array}{c}73.33 \% \\
(44.91-92.05)\end{array}$ & $\begin{array}{c}80.95 \% \\
(58.08-94.44)\end{array}$ & $\begin{array}{c}73.33 \% \\
(44.91-92.05)\end{array}$ & $\begin{array}{c}80.95 \% \\
(58.08-94.44)\end{array}$ & $\begin{array}{c}3.85 \\
(1.51-9.79)\end{array}$ & $77.78 \%$ \\
\hline Semiquantitative ${ }^{*}$ & $\begin{array}{c}100 \% \\
(78.03-100)\end{array}$ & $\begin{array}{c}90.48 \% \\
(69.58-98.55)\end{array}$ & $\begin{array}{c}88.24 \% \\
(63.52-98.20)\end{array}$ & $\begin{array}{c}100 \% \\
(82.20-100)\end{array}$ & $\begin{array}{c}10.50 \\
(2.81-39.24)\end{array}$ & $94.44 \%$ \\
\hline
\end{tabular}


TABLE 3. Semiquantitative ${ }^{99 m}$ Tc-MIBI Analysis Results

\begin{tabular}{|c|c|c|c|c|c|c|c|c|c|}
\hline \multicolumn{5}{|c|}{ Benign } & \multicolumn{5}{|c|}{ Malignant (carcinoma) } \\
\hline Case & Diagnosis & ER & DR & $\mathrm{RI}^{*}$ & Case & Diagnosis & ER & DR & $\mathrm{RI}^{\dagger}$ \\
\hline \multicolumn{10}{|c|}{ Nononcocytic lesions } \\
\hline $\mathrm{TH}-01$ & Goiter & 1.19 & 1.32 & 10.81 & TH-22 & Follicular & 1.83 & 2.70 & 47.52 \\
\hline TH-02 & Goiter & 2.72 & 2.25 & -17.28 & TH-23 & Follicular & 6.79 & 7.20 & 6.04 \\
\hline TH-03 & Goiter & 2.81 & 1.67 & -40.64 & TH-24 & Papillary & 0.58 & 0.68 & 17.97 \\
\hline TH-04 & Goiter & 1.09 & 0.87 & -20.41 & TH-25 & Papillary & 0.40 & 0.45 & 13.64 \\
\hline TH-05 & Goiter & 2.50 & 1.40 & -44.00 & TH-26 & Papillary & 2.01 & 2.53 & 25.82 \\
\hline TH-06 & Goiter & 0.84 & 0.72 & -14.91 & TH-27 & Papillary & 2.82 & 3.88 & 37.62 \\
\hline TH-07 & Goiter & 1.50 & 1.27 & -15.15 & TH-28 & Papillary & 2.68 & 3.00 & 12.15 \\
\hline TH-08 & Goiter & 5.53 & 4.67 & -15.66 & TH-29 & Papillary & 1.94 & 2.50 & 28.91 \\
\hline TH-09 & Goiter & 9.75 & 3.75 & -61.54 & TH-30 & Papillary & 1.43 & 1.88 & 31.92 \\
\hline $\mathrm{TH}-10$ & Adenoma & 2.32 & 4.67 & 101.25 & TH-31 & Papillary & 1.36 & 1.82 & 33.97 \\
\hline $\mathrm{TH}-11$ & Adenoma & 3.92 & 3.45 & -11.94 & TH-32 & Papillary & 1.49 & 1.68 & 12.73 \\
\hline $\mathrm{TH}-12$ & Adenoma & 1.96 & 0.78 & -60.25 & TH-33 & Papillary & 2.18 & 3.00 & 37.50 \\
\hline $\mathrm{TH}-13$ & Adenoma & 1.57 & 0.80 & -49.09 & TH-34 & Papillary & 1.24 & 1.23 & -0.81 \\
\hline $\mathrm{TH}-14$ & Adenoma & 1.80 & 0.83 & -53.70 & TH-35 & Papillary & 1.38 & 1.34 & -2.89 \\
\hline $\mathrm{TH}-15$ & Adenoma & 0.93 & 0.81 & -12.72 & TH-36 & Poorly differentiated & 2.62 & 4.50 & 71.76 \\
\hline TH-16 & Adenoma & 0.68 & 0.58 & -14.68 & & & & & \\
\hline $\mathrm{TH}-17$ & Adenoma & 1.35 & 1.18 & -12.78 & & & & & \\
\hline $\mathrm{TH}-18$ & Adenoma & 0.44 & 0.25 & -43.75 & & & & & \\
\hline TH-19 & Adenoma & 1.54 & 1.15 & -25.10 & & & & & \\
\hline TH-20 & Adenoma & 1.55 & 1.25 & -19.12 & & & & & \\
\hline TH-21 & Adenoma & 1.14 & 0.89 & -22.22 & & & & & \\
\hline \multicolumn{10}{|c|}{ Oncocytic lesions } \\
\hline TH-37 & Goiter & 3.00 & 3.25 & 8.33 & TH-48 & Papillary & 1.24 & 1.41 & 13.63 \\
\hline TH-38 & Goiter & 1.69 & 2.00 & 18.68 & $\mathrm{TH}-49$ & Papillary & 2.25 & 2.36 & 5.05 \\
\hline TH-39 & Goiter & 2.12 & 3.00 & 41.67 & TH-50 & Papillary & 0.70 & 1.00 & 43.33 \\
\hline TH-40 & Goiter & 0.39 & 0.55 & 39.39 & TH-51 & Insular & 3.10 & 3.84 & 23.84 \\
\hline TH-41 & Goiter & 1.20 & 1.42 & 17.88 & & & & & \\
\hline TH-42 & Adenoma & 1.17 & 1.65 & 41.02 & & & & & \\
\hline TH-43 & Adenoma & 2.90 & 5.67 & 95.29 & & & & & \\
\hline TH-44 & Adenoma & 2.68 & 2.07 & -22.67 & & & & & \\
\hline TH-45 & Adenoma & 4.00 & 3.00 & -25.00 & & & & & \\
\hline TH-46 & Adenoma & 2.23 & 4.17 & 86.78 & & & & & \\
\hline TH-47 & Adenoma & 1.76 & 1.96 & 11.65 & & & & & \\
\hline
\end{tabular}

*For benign nononcocytic lesions, all but 2 cases showed negative RI values. The 2 RI-positive cases had negative MRP1 apical expression (-). For benign oncocytic lesions, all but 2 cases showed positive RI values. The 2 RI-negative cases had positive MRP1 apical expression $(++)$.

${ }^{\dagger}$ For malignant nononcocytic lesions, all but 2 cases showed positive RI values. The 2 RI-negative cases had positive MRP1 apical expression (++). For malignant oncocytic lesions, all cases showed positive RI values and had negative MRP1 apical expression (-).

The intraobserver and interobserver correlation coefficients $(r)$ of ER values, with their related regression equations, were $r=0.996(y=0.9661 x+0.0501)$ and $r=0.983(y=1.0684 x-0.0536)$, respectively, whereas those of the DR values were $r=0.984(y=0.9822 x+$ $0.1695)$ and $r=0.956(y=0.9597 x+0.2107)$, respectively. The repeatability coefficients for early and delayed images were 0.283 and 0.523 , respectively.

No significant difference in ER values was observed between malignant and benign nononcocytic lesions, whereas significant differences were found for both DR and RI values $(P<0.05$ and $P<0.001$, respectively) in the same group.

No significant differences in the ER, DR, and RI indices were observed between malignant and benign oncocytic thyroid nodules.
No correlation was found between nodule size and RI value $(s=-0.098, \mathrm{CI}=-0.364$ to $0.182, P=0,486)$.

The RI cutoff corresponding to the highest accuracy for discriminating between benign and malignant nononcocytic thyroid lesions was -11.94 . Eleven of 15 nononcocytic nodules with an RI above this cutoff had a histologic result consistent with malignancy (Figs. 1A and 1B), whereas 17 of 21 lesions with a RI equal to or below this cutoff was histologically benign (Figs. 1C and 1D). For this threshold, the sensitivity and specificity were $100 \%$ (CI, 78.03-100) and $90.48 \%$ (CI, 69.58-98.55), respectively. The positive and negative predictive values were $88.24 \%$ (CI, 63.5298.20) and $100 \%$ (CI, 82.20-100), respectively. The diagnostic accuracy of the semiquantitative ${ }^{99 \mathrm{~m}} \mathrm{Tc}-\mathrm{MIBI}$ assay was $94.44 \%$, and the positive likelihood ratio was 10.50 
FIGURE 1. Thyroid 99mTc-MIBI scan. (A and B) Representative positive case (RI, 71.76) of solitary cold thyroid nodule in right lobe histologically diagnosed as nononcocytic solid/trabecular poorly differentiated carcinoma. (A) Early image with tracer accumulation in nodule (ER, 2.62). (B) Delayed image with tracer retention in lesion (DR, 4.50). (C and D) Representative negative case (RI, -40.64) of solitary cold thyroid nodule in left lobe that proved to be microfollicular goiter. (C) Early image with tracer increase uptake in nodule (ER, 2.81). (D) Delayed image with nearly complete tracer washout from nodule (DR, 1.67).
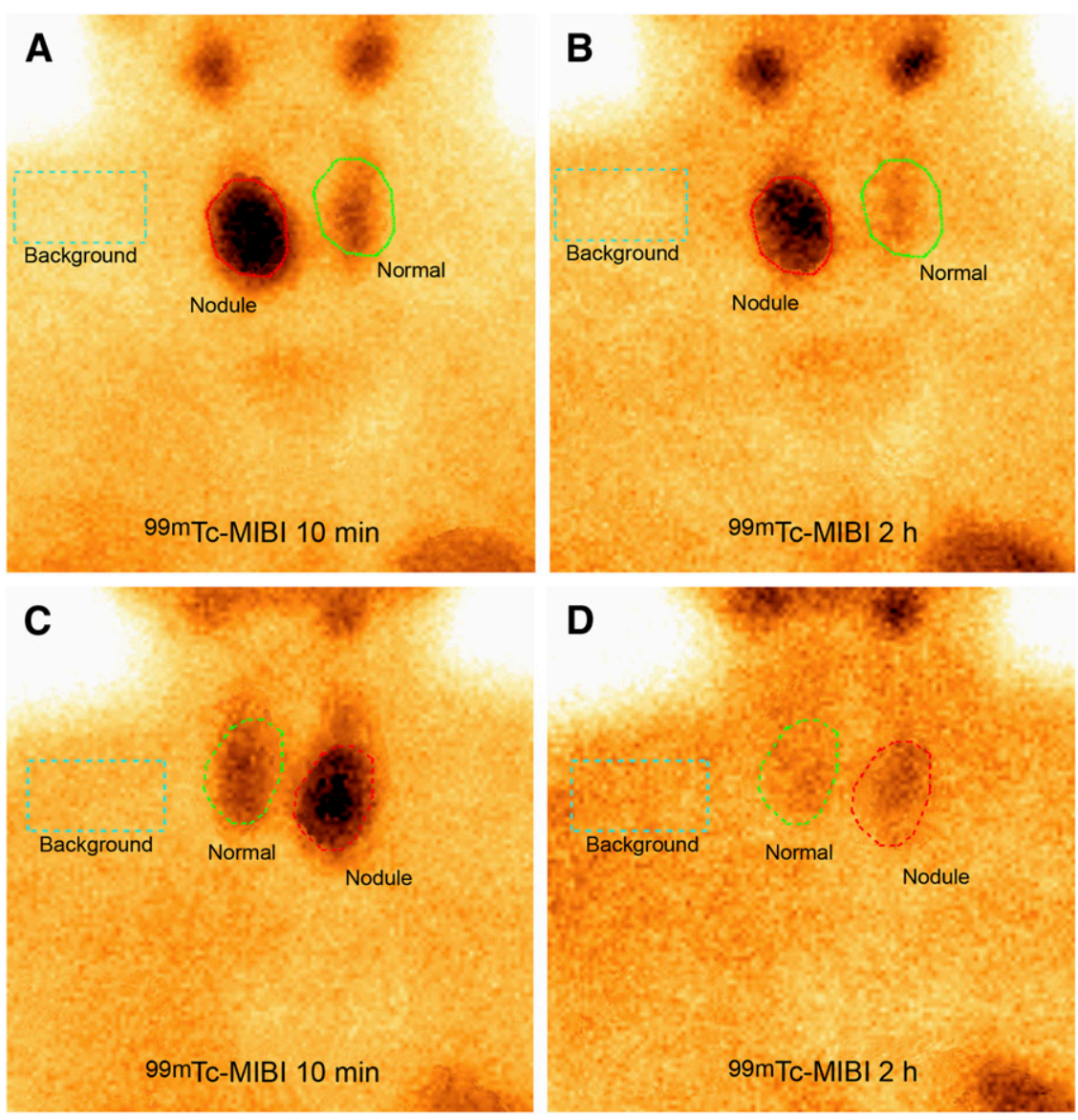

(CI, 2.81-39.24) (Table 2). The area under the receiver operating characteristic curve was 0.943 (CI, 0.811-0.991, $P=0.0001$ ), and SE was 0.044 .

\section{Multidrug Resistance Protein Expression}

No P-gp immunoreactivity was found in the follicular cells of either nononcocytic or oncocytic histologic specimens (Fig. 2A).

All but 3 cases ( 2 nononcocytic follicular adenomas and 1 nononcocytic follicular variant of papillary carcinoma) had a moderate or diffuse cytoplasmic immunoreaction for MRP1, with a granular pattern of staining (Figs. 2B, 2C, and 2D).

Fourteen of $21(67 \%)$ nononcocytic benign lesions showed apical membranous MRP1 staining in more then $25 \%$ of follicular cells (Fig. 2B), whereas 7 cases were either negative ( 4 samples) or only focally ( $\leq 25 \%$ of cells) positive (3 samples; 14\%) (Table 1).

All but 3 (80\%) (Fig. 2C) nononcocytic carcinomas (1 minimally invasive follicular carcinoma and 2 follicular variants of papillary carcinomas) were negative for MRP1 or showed only occasional cells with apical immunoreactivity (Table 1; Fig. 2D).

Only 4 cases ( 3 follicular adenomas and 1 microfollicular goiter; 27\%) of 15 oncocytic lesions had apical MRP1 expression in $25 \%-50 \%$ of follicular cells, whereas the remaining 11 cases (73\%) were negative or showed only occasional cells with apical immunoreactivity (Table 1).
Normal thyroid tissue surrounding tumor nodules was MRP1-negative or contained occasional positive cells. All nonfollicular cells were completely nonreactive.

99mTc-MIBI and MRP1 Membranous Apical Expression

Eighteen of 23 cases (78\%) with a negative RI value at semiquantitative ${ }^{99 \mathrm{~m}}$ Tc-MIBI analysis had apical membranous MRP1 staining in more then $25 \%$ of follicular cells (Figs. 2B and 2C), whereas 3 cases (13\%) were only focally ( $\leq 25 \%$ of cells) positive. The remaining 2 cases $(9 \%)$ were negative at the apical level.

All but 3 cases $(89 \%)$ with a positive RI value at semiquantitative ${ }^{99 \mathrm{~m}}$ Tc-MIBI analysis did not show apical MRP1 staining or showed only occasional cells with apical immunoreactivity (Fig. 2D). The 3 positive cases $(11 \%)$ presented MRP1 apical immunoreactivity in $25 \%-50 \%$ of follicular cells.

MRP1 membranous apical expression in follicular cells was significantly associated with negative ${ }^{99 \mathrm{~m}}$ Tc-MIBI RI values $(P<0.001)$.

A significant inverse correlation between the percentage of apical MRP1-positive follicular cells and negative values for RI was also detected ( $P<0.001,3$ degrees of freedom).

\section{DISCUSSION}

Today, fewer than $25 \%$ of nodules with indeterminate FNAB results (Thy3 [follicular neoplasm]) show malignant 

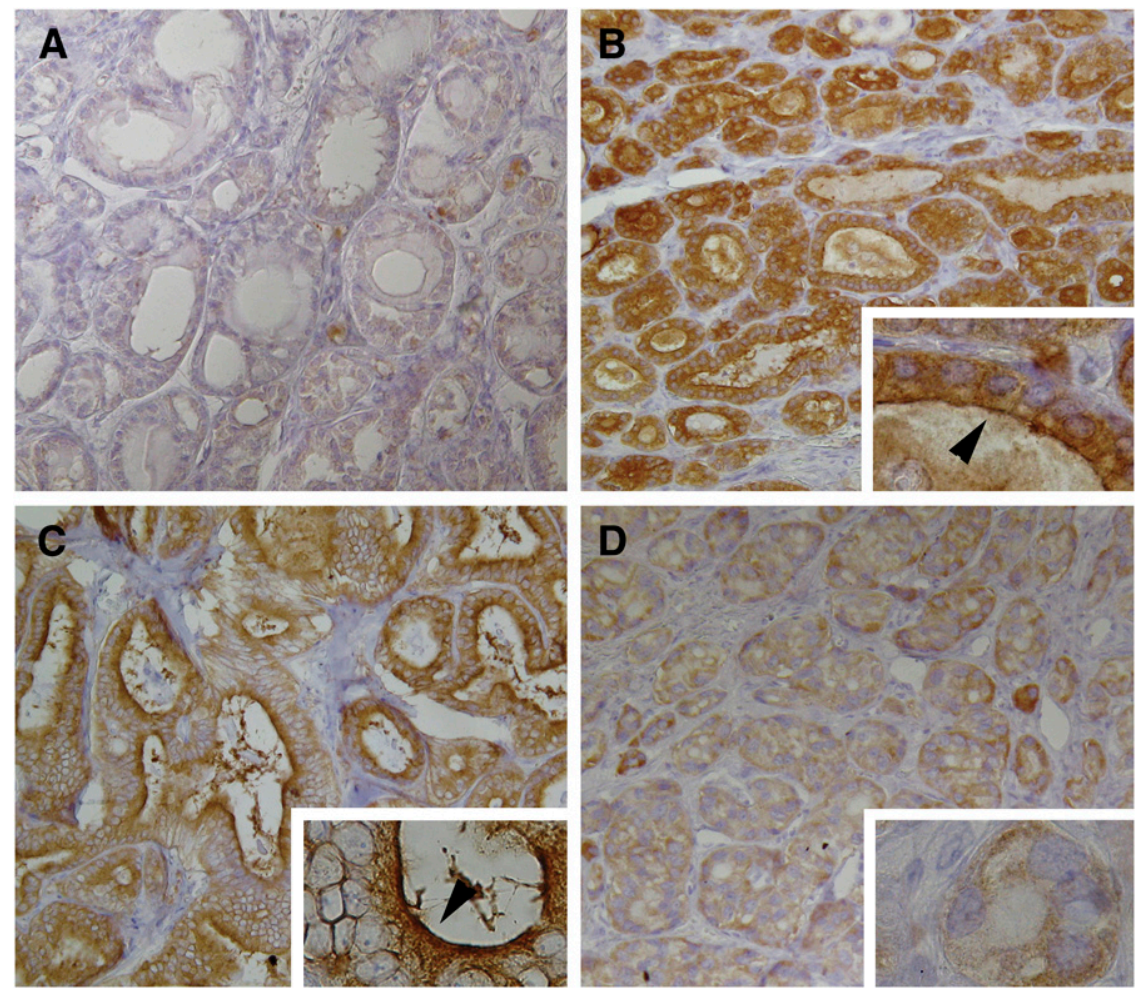

FIGURE 2. MDR1 expression in thyroid nodules with cytologic result of follicular neoplasm. (A) No P-gp immunoreactivity of follicular cells in nononcocytic emblematic case (follicular adenoma). (B and C) MRP1 membranous apical immunoexpression in case of nononcocytic microfollicular goiter (B) and in case of nononcocytic follicular variant of papillary carcinoma $(\mathrm{C})$, both with negative RI indices. Cytoplasmic immunoreactivity with granular pattern is seen. (D) No MRP1 membranous apical labeling in case of nononcocytic solid/trabecular papillary carcinoma with positive RI value. Cytoplasmic immunoreactivity with granular pattern is seen. Arrows show linear membranous apical immunoreactivity. Immunoperoxidase with Mayer solution counterstain was used (magnification, $\times 160$; insets: magnification, $\times 630)$.

features at histologic examination. Thus, most FNABs with indeterminate results are histologically benign, corresponding to unnecessary operations $(1,40)$.

Several authors have expressed the possibility that ${ }^{99 m}$ Tc-MIBI scintigraphy is a reliable adjunctive procedure for improving the diagnostic accuracy of thyroid fineneedle aspiration cytology (18-24). However, the overall published data on effectiveness in differentiating thyroid cancer from benign lesions are inconclusive, mostly because of low specificity (18).

Observations that ${ }^{99 \mathrm{~m}} \mathrm{Tc}-\mathrm{MIBI}$ scintigraphy is often positive in both benign and malignant oncocytic thyroid lesions $(22,25-27)$ prompted us to investigate whether its diagnostic accuracy could be increased when the nononcocytic thyroid follicular neoplasms were in advance separated (by cytologic examination) from the mitochondria-rich oncocytic ones. This study was prospectively conducted on a series of 51 cases of follicular neoplasms using both visual and semiquantitative ${ }^{99 \mathrm{~m}}$ Tc-MIBI analyses.

Hence, the first issue to solve in this work was to test the concordance between the cytologic definition of oncocytic follicular neoplasm and the corresponding postsurgical diagnoses, since a cytologic definition of oncocytic lesions (in terms of the number of oncocytic cells that should be present in a cytologic sample) is not provided in the literature. However, the cutoff of $50 \%$ oncocytic cells that we used in this study was able to correctly categorize all samples defined as oncocytic tumors on the surgical specimen.

A further matter of concern was the size of the nodule. In fact, taking into consideration the resolution limits of $\gamma$-cameras, we reasonably selected only patients with nodules measuring at least $10 \mathrm{~mm}$; no significant differences in nodule size were observed between positive and negative ${ }^{99 \mathrm{~m}} \mathrm{Tc}$ MIBI findings, although we could not investigate if lesions smaller than $1 \mathrm{~cm}$ had biologic or functional features potentially able to influence ${ }^{99 \mathrm{~m}} \mathrm{Tc}-\mathrm{MIBI}$ uptake and retention.

Our findings showed that visual and semiquantitative ${ }^{99 m}$ Tc-MIBI scintiscans were not specific enough to differentiate benign oncocytic lesions from malignant ones; most oncocytic lesions, both benign and malignant, were characterized by a significantly increased uptake or retention of ${ }^{99 \mathrm{~m}}$ Tc-MIBI at $2 \mathrm{~h}$, in agreement with other studies (22, 25-27). Therefore, we do not advise exposing patients to ${ }^{99 m}$ Tc-MIBI scintigraphy when the cytologic result is oncocytic follicular neoplasm.

As far as nononcocytic follicular neoplasms are concerned, semiquantitative ${ }^{99 \mathrm{~m}}$ Tc-MIBI scanning reached a diagnostic accuracy of $94.44 \%$, showing a better sensitivity and specificity than visual analysis (100\% and $73.33 \%$ vs. $90.48 \%$ and $80.95 \%$, respectively). However, these results are from relatively few patients and the semiquantitative analysis is at least partially an operator-dependent technique (e.g., region-of-interest-drawing phase) and depends on the instruments used, the imaging assays, and the peculiarities of the local patient population (22). For these reasons, it is important to state that our data have to be confirmed on a larger population and that each laboratory needs to set its own RI cutoff.

Notwithstanding these limitations, the promising results here obtained-sustained by good reproducibility and repeatability - prompt us to encourage the use of ${ }^{99 \mathrm{~m}} \mathrm{Tc}$ MIBI scintigraphy in clinical practice as an adjunct to both 
FNAB and immunocytochemical evaluation of thyroid tumor-associated molecules.

Taken together, our results provide useful suggestions for better diagnostic management of patients bearing thyroid nodules with indeterminate FNAB results, potentially reducing unnecessary operations.

In the case of cytologic diagnosis of oncocytic follicular neoplasms, we propose that galectin-3 and cytokeratin-19 should be evaluated as previously reported (8). Conversely, in the case of nononcocytic follicular neoplasms, semiquantitative ${ }^{99 \mathrm{~m}}$ Tc-MIBI scintigraphy could be performed when galectin-3 and HBME-1 are negative (Fig. 3). If the results for both markers (galectin-3 and HBME-1) and ${ }^{99 m}$ Tc-MIBI scanning are negative, the clinician should adopt a close follow-up program. In the instance of positive findings on molecular testing or ${ }^{99 \mathrm{~m}} \mathrm{Tc}-\mathrm{MIBI}$ scintigraphy, the patient should be referred for thyroidectomy (Fig. 3).

To accurately evaluate P-gp and MRP1 immunohistochemical expression in formalin-fixed and paraffin-embedded thyroid tissue samples, we followed some of the recommendations previously made by Beck et al. (41), such as the use of 2 or more standardized anti-P-gp and anti-MRP1 antibodies recognizing different epitopes, as well as the use of tissuespecific controls and different antigen retrieval methods to restore accessibility of the epitopes. To this purpose, we preliminarily tested C219 and JSB-1 clones to P-gp and QCRL-1 and MRPr1 clones to MRP1, using both ethylenediamine-tetraacetate and citrate pretreatments. All the antibodies recognize intracellular epitopes (42-44). Because they are internally binding, their epitope-binding ability is less sensitive to conformational changes, glycosylation status, and environmental effects (e.g., high salt in the medium) than is observed for externally binding antibodies. Therefore, C219, JSB-1, QCRL-1, and MRPr1 are well suited for immunostain- ing of formalin-fixed and paraffin-embedded materials (42), in which the P-gp/MRP may be degraded, and for immunostaining when other spurious variables may be present.

In our preliminary experimental setting, no differences in staining pattern were observed between C219 and JSB-1 in thyroid tissue samples (all samples were negative), but in control tissues C219 with ethylene-diamine-tetraacetate pretreatment was slightly more sensitive than JSB-1. Likewise, QCRL-1 and MRPr1 displayed an overlapping staining pattern in thyroid tissues; QCRL-1 showed a better-defined membranous pattern in follicular cells, with minimal nonspecific immunoreactivity (data not shown).

As far as the relationship between the ${ }^{99 \mathrm{~m}} \mathrm{Tc}-\mathrm{MIBI}$ images and the expression of P-gp and MRP1 in the corresponding thyroid nodular lesions is concerned, we found a significant inverse association between the RI values and MRP1 expression on apical plasma membranes of follicular cells. Our study also revealed that high MRP1 apical expression levels correlated significantly with low negative RI indices. Moreover, these results suggest that MRP1, but not P-gp, has a possible role as a transporter of ${ }^{99 \mathrm{~m}} \mathrm{Tc}-\mathrm{MIBI}$, contributing to the washout of radiopharmaceuticals in thyroid nodules.

\section{CONCLUSION}

Our findings suggest that semiquantitative ${ }^{99 m}$ Tc-MIBI scintigraphy could be an adjunctive presurgical method to investigate nononcocytic follicular lesions, predicting the malignant behavior of thyroid nodules that are indeterminate at FNAB and better orienting their clinical management. Finally, the good correlation between immunohistochemical apical expression of MRP1 and the scintigraphic findings supports the ${ }^{99 \mathrm{~m}} \mathrm{Tc}-\mathrm{MIBI}$ results and provides tissue information on the possible molecular mechanisms responsible for ${ }^{99 \mathrm{~m}}$ Tc-MIBI images in thyroid lesions.
FIGURE 3. Proposed decision tree for thyroid follicular neoplasms combining $99 \mathrm{~m} T \mathrm{Tc}-\mathrm{MIBI}$ scanning with molecular marker panel that we previously described (8). For cytologic diagnosis of oncocytic follicular neoplasm, we propose evaluation of galectin-3 and cytokeratin-19. Conversely, for nononcocytic follicular neoplasms, semiquantitative 99mTc-MIBI scintigraphy could be performed when galectin-3 and HBME-1 are negative. Negative results for both markers (galectin-3 and HBME-1) and for $99 \mathrm{mTC}-\mathrm{MIBI}$ scanning indicate that close follow-up is needed. If molecular testing or ${ }^{99 m}$ Tc-MIBI scintigraphy results are positive, patient should be referred for thyroidectomy. HBME-1 = cell surface mesothelial antigen HBME-1.

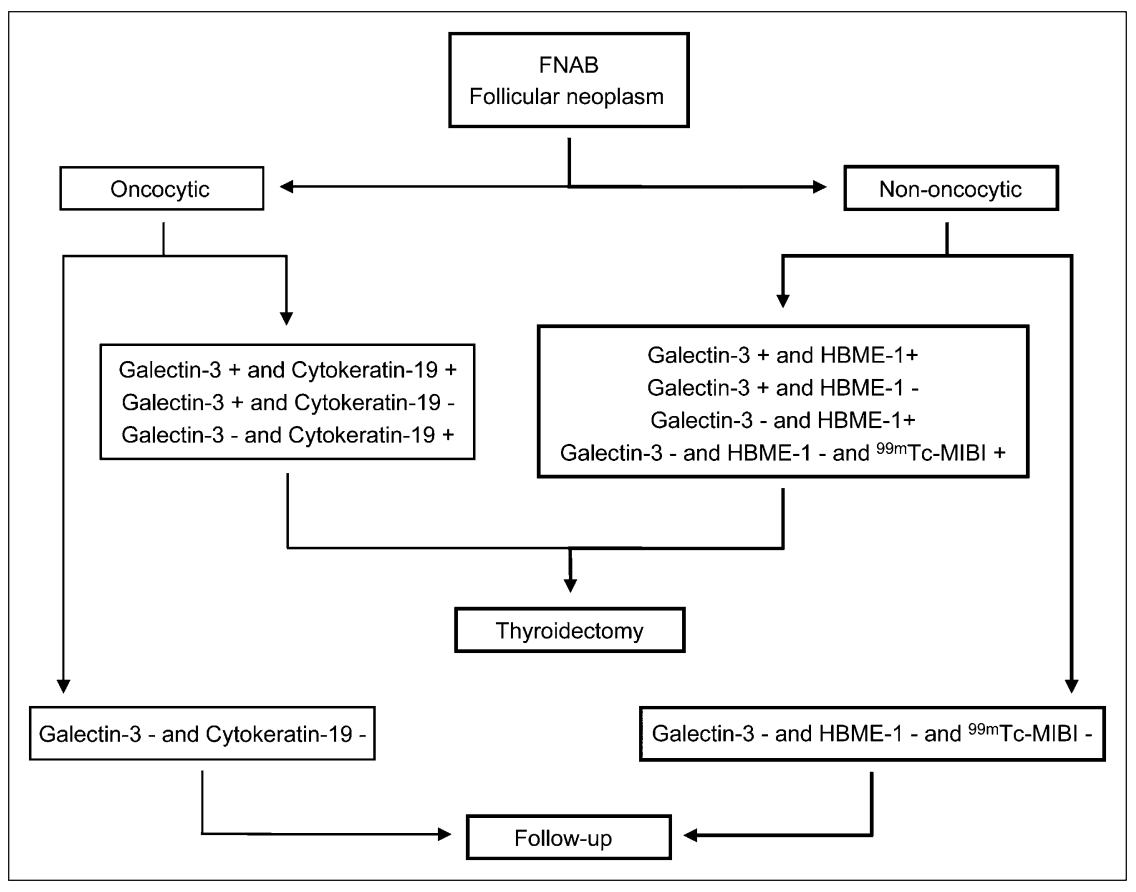




\section{ACKNOWLEDGMENTS}

We thank Dr. Carlotta Sacerdote (Unit of Cancer Epidemiology, University of Turin, Turin, Italy) for her invaluable help in the statistical analysis of these data, and Dr. Susanna Cappia (Division of Pathology, Department of Clinical and Biological Sciences, University of Turin, Turin, Italy) for her skilful technical assistance. This study was supported by grants from the Italian Ministry of University and Research (Rome, Italy) and in part by grants from Regione Piemonte (D.D. no. 12 of January 14, 2008, Turin, Italy).

\section{REFERENCES}

1. Schlumberger M, Pacini F. Thyroid Tumors. 2nd ed. Paris, France: Editions Nucléon; 2003:18-24.

2. Castro MR, Gharib H. Thyroid fine-needle aspiration biopsy: progress, practice, and pitfalls. Endocr Pract. 2003;9:128-136.

3. Baloch ZW, Fleisher S, LiVolsi VA, Gupta PK. Diagnosis of "follicular neoplasm": a gray zone in thyroid fine-needle aspiration cytology. Diagn Cytopathol. 2002;26:41-44.

4. Elsheikh TM, Asa SL, Chan JK, et al. Interobserver and intraobserver variation among experts in the diagnosis of thyroid follicular lesions with borderline nuclear features of papillary carcinoma. Am J Clin Pathol. 2008;130:736-744.

5. Lloyd RV, Erickson LA, Casey MB, et al. Observer variation in the diagnosis of follicular variant of papillary thyroid carcinoma. Am J Surg Pathol. 2004;28:1336-1340.

6. Kini S. Nodular goiter. In: Thyroid: Guides to Clinical Aspiration Biopsy. New York, NY: Igaku-Shoin; 1987:41-56.

7. Bartolazzi A, Orlandi F, Saggiorato E, et al. Galectin-3-expression analysis in the surgical selection of follicular thyroid nodules with indeterminate fine-needle aspiration cytology: a prospective multicentre study. Lancet Oncol. 2008;9:543549.

8. Saggiorato E, De Pompa R, Volante M, et al. Characterization of thyroid 'follicular neoplasms' in fine-needle aspiration cytological specimens using a panel of immunohistochemical markers: a proposal for clinical application. Endocr Relat Cancer. 2005;12:305-317.

9. Saggiorato E, Aversa S, Deandreis D, et al. Galectin-3: presurgical marker of thyroid follicular epithelial cell-derived carcinomas. $J$ Endocrinol Invest. 2004;27:311-317.

10. Bartolazzi A, Gasbarri A, Papotti M, et al. Application of an immunodiagnostic method for improving preoperative diagnosis of nodular thyroid lesions. Lancet. 2001;357:1644-1650.

11. Saggiorato E, Cappia S, De Giuli P, et al. Galectin-3 as a presurgical immunocytodiagnostic marker of minimally invasive follicular thyroid carcinoma. J Clin Endocrinol Metab. 2001;86:5152-5158.

12. Orlandi F, Saggiorato E, Pivano G, et al. Galectin-3 is a presurgical marker of human thyroid carcinoma. Cancer Res. 1998;58:3015-3020.

13. Carpi A, Naccarato AG, Iervasi G, et al. Large needle aspiration biopsy and galectin-3 determination in selected thyroid nodules with indeterminate FNAcytology. Br J Cancer. 2006;95:204-209.

14. Asa SL. The role of immunohistochemical markers in the diagnosis of follicularpatterned lesions of the thyroid. Endocr Pathol. 2005;16:295-309.

15. Volante M, Bozzalla-Cassione F, DePompa R, et al. Galectin-3 and HBME1 expression in oncocytic cell tumors of the thyroid. Virchows Arch. 2004;445:183-188.

16. Liu YY, Morreau H, Kievit J, Romijn JA, Carrasco N, Smit JW. Combined immunostaining with galectin-3, fibronectin-1, CITED-1, Hector Battifora mesothelial-1, cytokeratin-19, peroxisome proliferator-activated receptor\{gamma , and sodium/iodide symporter antibodies for the differential diagnosis of non-medullary thyroid carcinoma. Eur J Endocrinol. 2008;158:375-384.

17. Prasad ML, Pellegata NS, Huang Y, Nagaraja HN, de la Chapelle A, Kloos RT. Galectin-3, fibronectin-1, CITED-1, HBME1 and cytokeratin-19 immunohistochemistry is useful for the differential diagnosis of thyroid tumors. Mod Pathol. 2005;18:48-57.

18. Hurtado-Lopez LM, Martinez-Duncker C. Negative MIBI thyroid scans exclude differentiated and medullary thyroid cancer in $100 \%$ of patients with hypofunctioning thyroid nodules. Eur J Nucl Med Mol Imaging. 2007;34:1701-1703.

19. Hurtado-Lopez LM, Arellano-Montano S, Torres-Acosta EM, et al. Combined use of fine-needle aspiration biopsy, MIBI scans and frozen section biopsy offers the best diagnostic accuracy in the assessment of the hypofunctioning solitary thyroid nodule. Eur J Nucl Med Mol Imaging. 2004;31:1273-1279.

20. Sharma R, Mondal A, Shankar LR, et al. Differentiation of malignant and benign solitary thyroid nodules using 30- and 120-minute tc-99m MIBI scans. Clin Nucl Med. 2004;29:534-537.

21. Sehgal AK, Sathekge MM, Mageza RB, Modiba MC. TC99m MIBI scintigraphy in well-differentiated thyroid carcinoma. Cent Afr J Med. 2001;47:97-102.

22. Erdil TY, Ozker K, Kabasakal L, et al. Correlation of technetium-99m MIBI and thallium-201 retention in solitary cold thyroid nodules with postoperative histopathology. Eur J Nucl Med. 2000;27:713-720.

23. Sathekge MM, Mageza RB, Muthuphei MN, Modiba MC, Clauss RC. Evaluation of thyroid nodules with technetium-99m MIBI and technetium-99m pertechnetate. Head Neck. 2001;23:305-310.

24. Mezosi E, Bajnok L, Gyory F, et al. The role of technetium-99m methoxyisobutylisonitrile scintigraphy in the differential diagnosis of cold thyroid nodules. Eur $J$ Nucl Med. 1999;26:798-803.

25. Boi F, Lai ML, Deias C, et al. The usefulness of ${ }^{99 \mathrm{~m}} \mathrm{Tc}$-sestaMIBI scan in the diagnostic evaluation of thyroid nodules with oncocytic cytology. Eur $J$ Endocrinol. 2003;149:493-498.

26. Vattimo A, Bertelli P, Cintorino M, Burroni L, Volterrani D, Vella A. Identification of Hurthle cell tumor by single-injection, double-phase scintigraphy with technetium-99m-sestamibi. J Nucl Med. 1995;36:778-782.

27. Chamnanrabiabkij E, Welch A, Jayapaul MK, Perros P. Detection of Hurthle cell carcinoma using sestamibi. Thyroid. 2008;18:575-576.

28. Moretti JL, Hauet N, Caglar M, Rebillard O, Burak Z. To use MIBI or not to use MIBI? That is the question when assessing tumour cells. Eur J Nucl Med Mol Imaging. 2005;32:836-842.

29. Piwnica-Worms DP, Kronauge JF, LeFurgey A, et al. Mitochondrial localization and characterization of 99Tc-SESTAMIBI in heart cells by electron probe X-ray microanalysis and 99Tc-NMR spectroscopy. Magn Reson Imaging. 1994;12:641-652.

30. Chernoff DM, Strichartz GR, Piwnica-Worms D. Membrane potential determination in large unilamellar vesicles with hexakis(2-methoxyisobutylisonitrile)technetium(I). Biochim Biophys Acta. 1993;1147:262-266.

31. Zhou SF. Structure, function and regulation of P-glycoprotein and its clinical relevance in drug disposition. Xenobiotica. 2008;38:802-832.

32. Altenberg GA. Structure of multidrug-resistance proteins of the ATP-binding cassette (ABC) superfamily. Curr Med Chem Anticancer Agents. 2004;4: $53-62$.

33. Sharom FJ. ABC multidrug transporters: structure, function and role in chemoresistance. Pharmacogenomics. 2008;9:105-127.

34. Hendrikse NH, Franssen EJ, van der Graaf WT, et al. ${ }^{99 \mathrm{~m}} \mathrm{Tc}$-sestamibi is a substrate for P-glycoprotein and the multidrug resistance-associated protein. $\mathrm{Br} \mathrm{J}$ Cancer. 1998;77:353-358.

35. Hipfner DR, Deeley RG, Cole SP. Structural, mechanistic and clinical aspects of MRP1. Biochim Biophys Acta. 1999;1461:359-376.

36. British Thyroid Association, Royal College of Physicians. Guidelines for the management of thyroid cancer. In: Perros P, ed. Report of the Thyroid Cancer Guidelines Update Group. 2nd ed. London, U.K.: Royal College of Physicians; 2007:9-10.

37. DeLellis R, Lloyd R, Heitz P, Eng C. Pathology and genetics of tumours of the endocrine organs. In: Kleihues P, Sobin L, eds. World Health Organization Classification of Tumours. Lyon, France: IARC; 2004:49-133.

38. Greenspan BS, Brown ML, Dillehay GL, et al. The Society of Nuclear Medicine Procedure Guideline for Parathyroid Scintigraphy. Version 3.0. Reston, VA: Society of Nuclear Medicine; 2004.

39. Bland JM, Altman DG. Statistical methods for assessing agreement between two methods of clinical measurement. Lancet. 1986;1:307-310.

40. Hegedus L, Bonnema SJ, Bennedbaek FN. Management of simple nodular goiter: current status and future perspectives. Endocr Rev. 2003;24:102132.

41. Beck WT, Grogan TM, Willman CL, et al. Methods to detect P-glycoproteinassociated multidrug resistance in patients' tumors: consensus recommendations. Cancer Res. 1996;56:3010-3020.

42. Scheffer GL, Kool M, Heijn M, et al. Specific detection of multidrug resistance proteins MRP1, MRP2, MRP3, MRP5, and MDR3 P-glycoprotein with a panel of monoclonal antibodies. Cancer Res. 15 2000;60:5269-5277.

43. Scheper RJ, Bulte JW, Brakkee JG, et al. Monoclonal antibody JSB-1 detects a highly conserved epitope on the P-glycoprotein associated with multi-drugresistance. Int J Cancer. 1988;42:389-394.

44. Hipfner DR, Gao M, Scheffer G, Scheper RJ, Deeley RG, Cole SP. Epitope mapping of monoclonal antibodies specific for the 190-kDa multidrug resistance protein (MRP). Br J Cancer. 1998;78:1134-1140. 